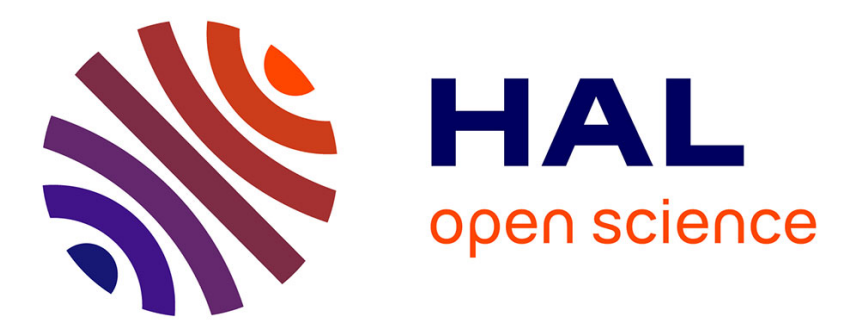

\title{
Realistic prediction of outage probability and confidence interval of BER for indoor radio communications
}

\author{
Meiling Luo, Guillaume Villemaud, Jean-Marie Gorce, Jie Zhang
}

\section{To cite this version:}

Meiling Luo, Guillaume Villemaud, Jean-Marie Gorce, Jie Zhang. Realistic prediction of outage probability and confidence interval of BER for indoor radio communications. EUCAP 2014, Apr 2014, The Hague, Netherlands. pp.2105 - 2109, 10.1109/EuCAP.2014.6902223 . hal-01090704

\section{HAL Id: hal-01090704 https://hal.inria.fr/hal-01090704}

Submitted on 4 Dec 2014

HAL is a multi-disciplinary open access archive for the deposit and dissemination of scientific research documents, whether they are published or not. The documents may come from teaching and research institutions in France or abroad, or from public or private research centers.
L'archive ouverte pluridisciplinaire HAL, est destinée au dépôt et à la diffusion de documents scientifiques de niveau recherche, publiés ou non, émanant des établissements d'enseignement et de recherche français ou étrangers, des laboratoires publics ou privés. 


\title{
Realistic Prediction of Outage Probability and Confidence Interval of BER for Indoor Radio Communications
}

\author{
Meiling Luo ${ }^{1}$, Guillaume Villemaud ${ }^{1}$, Jean-Marie Gorce ${ }^{1}$, Jie Zhang ${ }^{2}$ \\ ${ }^{1}$ CITI, INSA-Lyon, INRIA, Université de Lyon, 69621 Villeurbanne, France \\ ${ }^{2}$ Department of Electronic and Electrical Engineering, University of Sheffield, Sheffield S1 3JD, U.K.
}

\begin{abstract}
Outage probability and Bit Error Rate (BER) are both important link quality parameters for wireless communication systems. In this paper, the error in $\mathrm{dB}$ between the MultiResolution Frequency-Domain ParFlow (MR-FDPF) model and the channel measurement is first modeled to follow the normal distribution which is verified by performing the Lilliefors goodness of fit test. Then a realistic BER map as well as its confidence interval can be predicted. In addition, the normally distributed error in $\mathrm{dB}$ can be regarded as a kind of lognormal shadowing introduced by the MR-FDPF model due to, for instance, the inaccurate scenario modeling. Then a realistic outage probability can be computed by taking into account both the large scale lognormal shadowing and the small scale Nakagami-m fading.
\end{abstract}

Index Terms - outage probability, BER, indoor radio propagation.

\section{INTRODUCTION}

Radio link quality prediction is imperative for the efficiency of wireless communication systems. For instance, many adaptive techniques [1], such as adaptive modulation and coding (AMC) scheme or optimal power allocation, need the link quality information to dynamically adapt their scheme. Thus, the accuracy of the radio link quality prediction is crucial for wireless communication systems since it may impact the higher layer strategies.

Outage probability [2] and Bit Error Rate (BER) are two important radio link quality parameters. The Bit Error Rate (BER) is the ratio of the number of the received error bits to the total number of the transferred bits. The outage probability is the probability that the outage occurs, i.e., when the received SNR falls below a threshold required for an accepted level of performance or equivalently when the BER exceeds a corresponding threshold due to fading. Therefore, a realistic prediction of the outage probability and BER is of importance for wireless communication systems.

In this paper, we will predict the outage probability and BER based on the Multi-Resolution Frequency-Domain ParFlow (MR-FDPF) model [3]-[7] which will be detailed in section II. For a simulation scenario, the MR-FDPF model can provide an electric field map or a coverage map (mean power map) on a pixel level basis. Even though the MR-FDPF model has a high level of accuracy as a deterministic radio propagation model, it still always has a Root Mean Square Error (RMSE) when compared to channel measurements. This error can be modeled to follow the normal distribution which can be verified by performing the Lilliefors goodness of fit test. Then this normally distributed error in $\mathrm{dB}$ can be used to evaluate the confidence interval of the predicted BER. Moreover, after this normally distributed error in $\mathrm{dB}$ is regarded as a kind of lognormal shadowing, a realistic outage probability can be computed by taking into account both the large scale shadow fading and the small scale Nakagami-m fading.

The rest of the paper is organized as follows. Section II gives a brief introduction of the MR-FDPF model, and then in section III, the BER and its confidence interval will be predicted. Finally, the prediction of the outage probability will be detailed in section IV, followed by the conclusion in section V.

\section{THE MR-FDPF MODEL}

The MR-FDPF model is a deterministic Finite-Difference Time-Domain (FDTD)-like model [3]-[7]. Its main difference from the traditional FDTD model is that the MR-FDPF model works in the frequency domain instead of the time domain. In the time domain, the FDTD model needs a sufficient number of time iterations to reach the steady state, whereas, in the frequency domain, the MR-FDPF model can solve the steady state problem directly. Moreover, the steady state problem in the frequency domain is a linear matrix inversion problem which allows the MR-FDPF model to introduce a MultiResolution (MR) structure to further reduce the computational complexity. For a specific propagation scenario, the MR-FDPF model should first discretize it into a grid-based structure (we would like to call the smallest grids pixels). Based on this gridbased structure, the MR-FDPF model can provide an electric field map, and thus also a coverage map for the propagation scenarios. In Fig. 1, a radio coverage map provided by the MR-FDPF model is presented as an example, obtained at 2.45 $\mathrm{GHz}$ with $23 \mathrm{dBm}$ transmit power for the Stanford University's scenario [8].

Even if a lot has been done to reduce the computational complexity in the MR-FDPF model, the required computational load is still very high. Due to this reason, the MRFDPF model is mainly restricted to 2 Dimensional (2D) and to small propagation scenarios, such as the indoor radio propagation scenarios. Like any other radio propagation model, a calibration process is imperative for the MR-FDPF model 


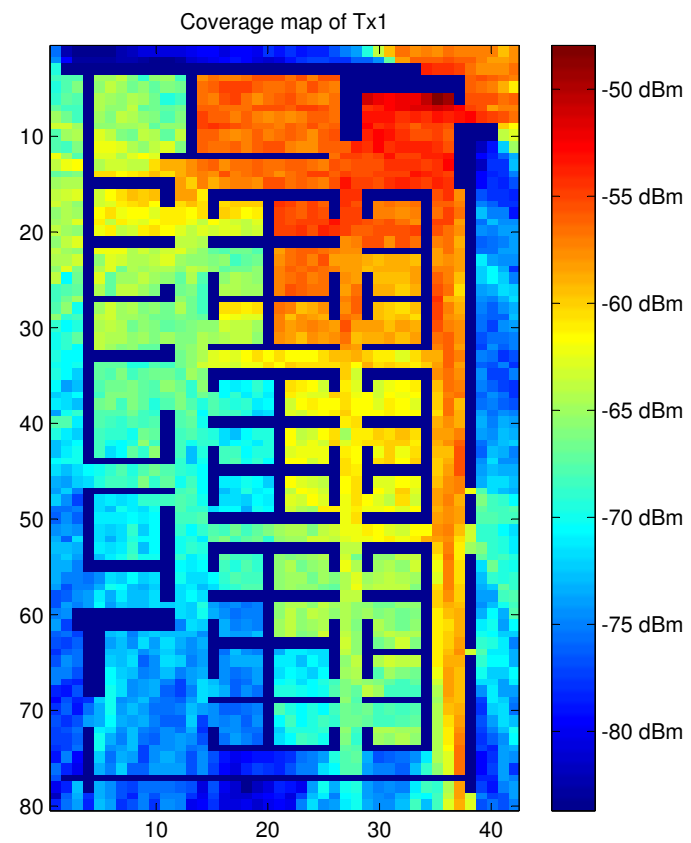

Fig. 1. Coverage map of Tx 1 predicted with the MR-FDPF model at 2.45 $\mathrm{GHz}$.

since the properties of materials, e.g. walls in the simulated scenarios are never known exactly. For the MR-FDPF model, the calibration process is done in two steps. The first step is to estimate the constant offset as follows

$$
\Delta \Psi=\frac{1}{N} \sum_{k=1}^{N}\left(\Psi_{m e s}(k)-\Psi_{\text {sim }}(k)\right)
$$

where $\Psi_{\text {mes }}(k)$ and $\Psi_{\text {sim }}(k)$ are the mean powers from the channel measurement and the MR-FDPF simulation, respectively, and $N$ is the total number of samples. A constant offset always exists because of the numerical sources used in the MR-FDPF model, compared to the real transmitters in reality. The second step of the calibration process is to estimate the absorption coefficient of the air $a_{a i r}$, the refraction index and the normalized absorption coefficient of materials $\left(n_{\text {mat }}, a_{\text {mat }}\right)$ by minimizing the cost-function $Q$ defined by the RMSE between the channel measurement and the prediction

$$
Q=R M S E=\sqrt{\frac{1}{N} \sum_{k=1}^{N}\left\|\Psi_{\text {mes }}(k)-\Psi_{\text {pred }}(k)\right\|^{2}}
$$

where

$$
\Psi_{\text {pred }}(k)=\Psi_{\text {sim }}(k)+\Delta \Psi
$$

are the mean powers from the prediction.

Since no radio propagation models can be totally perfect, a RMSE always exists between the MR-FDPF simulation and the channel measurement. For the simulation, this RMSE can be regarded as the error introduced by the MR-FDPF model due to, for instance, the inaccurate scenario modeling. In the next section, we will see that this error can be modeled to follow the normal distribution.

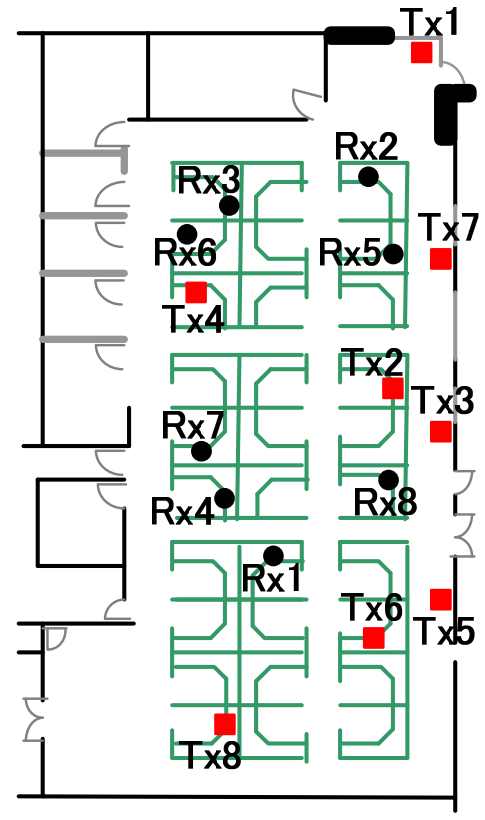

Fig. 2. The Stanford University's measurement scenario.

\section{BER AND CONFIDENCE INTERVAL}

Since we need a measurement campaign to calibrate our MR-FDPF model, we will introduce the measurement scenario and measurement setup first in this section. Here, we use the measurement campaign from Stanford University [8] to calibrate the MR-FDPF model, and specifically it corresponds to the "I2I stationary" scenario measurement therein.

\section{A. The office scenario}

This is a typical office scenario which is about $16 \mathrm{~m} \times 34 \mathrm{~m}$. It consists of 30 cubicles and 7 small separated rooms. There are totally 8 transmitters and 8 receivers which are distributed in the office as shown in Fig. 2. All of them are equipped with omnidirectional antennas and are fixed in their locations during the measurement. There are mainly four materials used in this office, i.e., concrete for the main walls, plaster for the internal walls, glass for the external glass wall and wood for the cubicles located in the central part of the office.

\section{B. Measurement setup}

This is a 8 × 8 Multiple-Input Multiple-Output (MIMO) channel measurement conducted with a RUSK MEDAV channel sounder [9]. The center frequency is $2.45 \mathrm{GHz}$ with a bandwidth of $70 \mathrm{MHz}$. In the measurement, 120 time blocks covering a total time of 32 seconds and 220 frequency bins were recorded.

\section{Calibration}

The calibration is performed with the measurement data from the links between the Tx1 and all the 8 Rxs. The measurement data used to perform the calibration are taken only for the center frequency of $2.45 \mathrm{GHz}$, but are averaged over the 120 time blocks since it is a stationary measurement. 
TABLE I

PARAMETER VALUES OF MATERIALS OPTIMIZED B Y THE CALIBRATION

\begin{tabular}{|c|c|c|}
\hline & $n_{\text {mat }}$ & $a_{\text {mat }}$ \\
\hline air & 1.0 & 0.9999997 \\
\hline absorbant & 1.0 & 0.96879673 \\
\hline wood & 2.3888888 & 0.9999999 \\
\hline plaster & 1.5 & 0.9999999 \\
\hline concrete & 5.4 & 0.9999999 \\
\hline glass & 2.0438957 & 0.9999999 \\
\hline
\end{tabular}

The obtained parameter values of materials are listed in Table. I. These parameters are configured to run the MR-FDPF simulation at $2.45 \mathrm{GHz}$. Thus, we can compute the offset and the RMSE between the simulation and measurement for the 8 links from Tx1 according to (1) and (2): offset $=-62.1 \mathrm{dBm}$, $\mathrm{RMSE}=4.4 \mathrm{~dB}$.

\section{The Nakagami-m fading}

The Nakagami-m fading is given as follows [10]

$$
P_{\alpha}(\alpha)=\frac{2 m^{m} \alpha^{2 m-1}}{\Omega^{m} \Gamma(m)} \exp \left(-\frac{m \alpha^{2}}{\Omega}\right), \quad \alpha \geq 0
$$

where $\Omega=\mathbb{E}\left(\alpha^{2}\right)$ is the average power of the fading and $m$ is called the Nakagami-m fading parameter. Here, we model the small scale fading to be the Nakagami-m fading since the Nakagami-m fading model can describe a wide range of small scale fading phenomena. For instance, it includes the Rayleigh fading as a special case when $m=1$ and includes the Rice fading when $m>1$.

For the estimation of the $m$ parameter of the Nakagami$m$ fading, the Greenwood's method can be adopted [7] [11], which can be briefly stated as follows

$$
\hat{m}=\left\{\begin{array}{l}
\frac{0.5000876+0.1648852 y-0.0544274 y^{2}}{y} \\
0<y \leq 0.5772 \\
\frac{8.898919+9.059950 y+0.9775373 y^{2}}{y\left(17.79728+11.968477 y+y^{2}\right)} \\
0.5772<y<17
\end{array}\right.
$$

where $y=\ln \left(\hat{\mu}_{2} / G\right), \ln (\cdot)$ is the natural logarithm operator, $\hat{\mu}_{2}$ denotes the arithmetic mean of sample power, i.e. $\hat{\mu}_{2}=$ $\frac{1}{N} \sum_{n=1}^{N} x_{n}^{2}$, and $G$ denotes the geometric mean of sample power $G=\left(\prod_{n=1}^{N} x_{n}^{2}\right)^{1 / N}$.

Fig. 3 shows the estimated $m$ parameter map of the Nakagami-m fading. More details about how to obtain this $m$ parameter map can be found in [7]. In this figure, each $m$ parameter is estimated over a local area with dimensions of $23 \times 23$ pixels. Moreover, the grey points denote the locations where the estimated $m$ parameter has failed the KolmogorovSmirnov test [12].

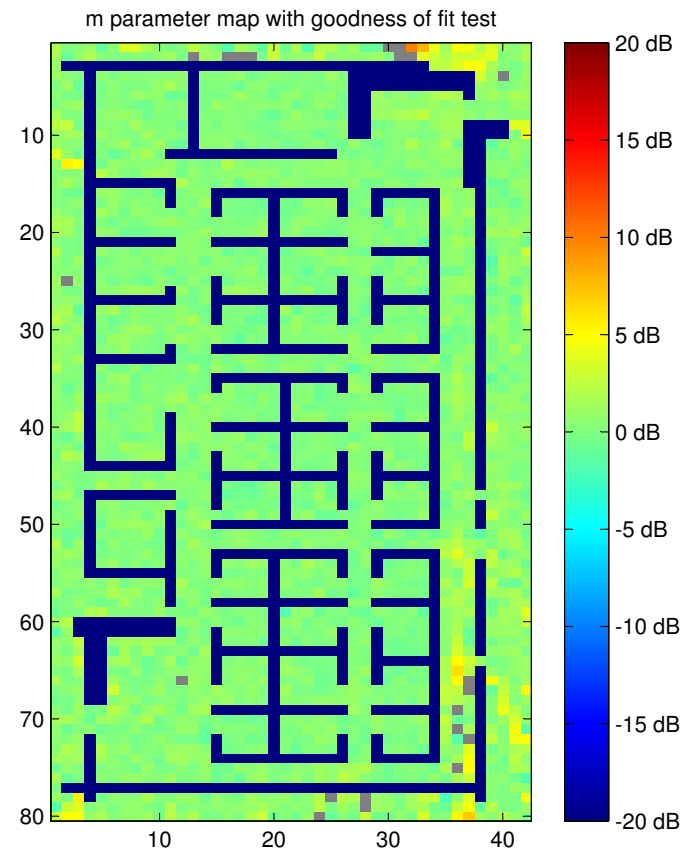

Fig. 3. The m parameter map by Greenwood's method with goodness of fit test.

\section{E. The BER and its confidence interval}

The BER for the M-PSK modulations over fading channels can be computed according to [7] [13]

$$
\begin{aligned}
& P_{b: \text { fading }}(E) \cong \frac{2}{\max \left(\log _{2} M, 2\right)} . \\
& \sum_{i=1}^{\max \left(\frac{M}{4}, 1\right)} \frac{1}{\pi} \int_{0}^{\pi / 2} M_{\gamma}\left(-\frac{\log _{2} M}{\sin ^{2} \theta} \sin ^{2} \frac{(2 i-1) \pi}{M}\right) d \theta
\end{aligned}
$$

and the BER for the M-QAM modulations over fading channels can be computed according to

$$
\begin{aligned}
P_{b: \text { fading }}(E) \cong \frac{4}{\log _{2} M}\left(1-\frac{1}{\sqrt{M}}\right) . \\
\quad \sum_{i=1}^{\sqrt{M} / 2} \frac{1}{\pi} \int_{0}^{\pi / 2} M_{\gamma}\left(-\frac{(2 i-1)^{2} \cdot 3 \cdot \log _{2} M}{2 \sin ^{2} \theta \cdot(M-1)}\right) d \theta
\end{aligned}
$$

where the $M_{\gamma}(\cdot)$ denotes the Moment Generating Function (MGF) associated with a specific fading.

From the above equations, we know that the BER over fading channels is the function of the MGF associated with the fading. For example, the MGF of the Nakagami-m fading is

$$
M_{\gamma}(s)=\left(1-\frac{s \cdot \bar{\gamma}}{m}\right)^{-m}
$$

where $m$ is the parameter of the Nakagami-m fading and $\bar{\gamma}$ is the average SNR. Thus, when provided with the $m$ parameter map in the previous subsection, we can easily compute the BER for the Nakagami-m fading channels. In Fig. 4, we present the predicted BER map for 16QAM from Tx1 as an example. In this figure, the transmit power is $23 \mathrm{dBm}$ and the 


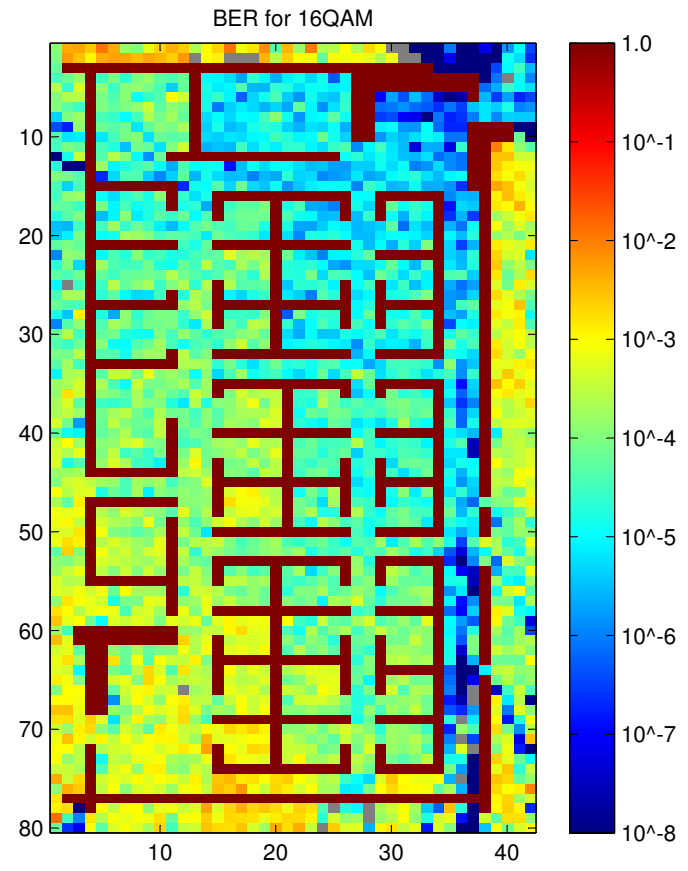

Fig. 4. BER for 16QAM.

thermal noise is considered over the bandwidth of 3.5 MHz. In this figure, as the same for the $m$ parameter map, each BER is computed over a local area with dimensions of $23 \times 23$ pixels. Here we also leave those points grey where the estimated $m$ parameter has failed the Kolmogorov-Smirnov test.

As stated in the subsection III-C, the obtained RMSE after calibration is $4.4 \mathrm{~dB}$. We perform a Lilliefors goodness of fit test at the 5\% significance level for a normal distribution to the error between the simulation and measurement. The Lilliefors goodness of fit test can not reject the null hypothesis that the samples come from a normal distribution, which means that the error can be modeled to follow a normal distribution in $\mathrm{dB}$. Therefore, the confidence interval of the signal mean power can be computed and thus that of the BER.

The confidence interval of the sample mean $\bar{X}$ for a normally distributed population with unknown mean and unknown standard deviation can be calculated according to the Student's t-distribution as follows [14]

$$
\left[\bar{X}-\frac{t S}{\sqrt{N}}, \bar{X}+\frac{t S}{\sqrt{N}}\right]
$$

where $N$ is the number of samples, $t$ is the critical value for the Student's t-distribution with $N-1$ degrees of freedom,

$$
\bar{X}=\left(X_{1}+X_{2}+\cdots X_{N}\right) / N
$$

and

$$
S=\sqrt{\frac{1}{N-1} \sum_{i=1}^{N}\left(X_{i}-\bar{X}\right)^{2}}
$$

is the sample standard deviation. By comparing (2) and (11), it is easy to know that the sample standard deviation $S$ can be computed from the RMSE by

$$
S=\sqrt{\frac{N}{N-1}} \cdot R M S E
$$

Thus, the confidence interval can be computed directly from the RMSE.

In this section, we studied how to predict the BER. Meanwhile, we investigated the confidence interval of the predicted BER by considering the error between the MR-FDPF model and the channel measurement. In the next section, the error between the MR-FDPF model and the channel measurement will be further considered in order to achieve a realistic prediction of the outage probability.

\section{Outage Probability}

As stated in the section III, the error between the MR-FDPF model and the channel measurement in $\mathrm{dB}$ can be modeled to follow a normal distribution. Thus, this normally distributed error in $\mathrm{dB}$ can be regarded as a kind of lognormal shadowing introduced by the inaccurate scenario modeling. As we know, in the multipath radio propagation, besides the large scale shadow fading, there exists also the small scale Nakagami-m fading. Thus, in order to be accurate, here we should take into account both the large scale shadow fading and the small scale Nakagami-m fading in order to compute the outage probability.

The outage probability over lognormal shadowed Nakagami-m fading channels can be computed according to [15]

$$
P=\int_{0}^{\infty} G\left(S_{\min }, m, \frac{\Omega}{m}\right) \frac{4.3429}{\Omega \sqrt{2 \sigma^{2}}} e^{-\frac{\left[10 \log _{10} \Omega-\mu\right]}{2 \sigma^{2}}} d \Omega
$$

where $S_{\min }$ is the minimum signal power required, $\mu$ (in $\mathrm{dB}$ ) and $\sigma$ (in $\mathrm{dB}$ ) are the mean and the standard deviation of $10 \log _{10} \Omega$, respectively, and $G(\cdot)$ is the Gamma cumulative distribution function defined as

$$
G\left(S_{\min }, m, \frac{\Omega}{m}\right)=\int_{0}^{\sqrt{S_{\min }}} \frac{2 m^{m}}{\Gamma(m) \Omega^{m}} x^{2 m-1} e^{-m \frac{x^{2}}{\Omega}} d x
$$

which can be directly implemented by the command gamcdf in Matlab [16].

We implement the formula (13) by Matlab to compute the outage probability for each local area mentioned above. Then we can obtain the predicted outage probability map for each modulation scheme since we already have the $m$ parameter map and the BER map available from the work of previous sections. In Fig. 5 and Fig. 6, we present the predicted outage probability maps from Tx1 for the 16QAM and 64QAM, respectively. In these figures, the $S_{\min }$ is set to the minimum signal power which satisfies the BER of $10^{-3}$. From these two figures, we can see that the 64QAM has a larger outage probability than the 16QAM since it requires a higher SNR than 16QAM in order to reach the same performance in terms of BER. 


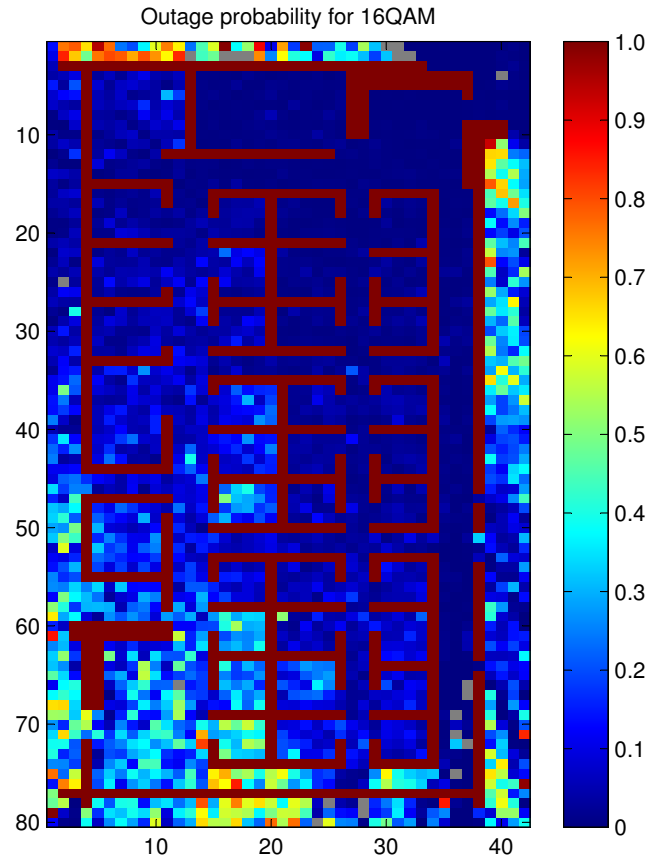

Fig. 5. Outage probability for 16QAM.

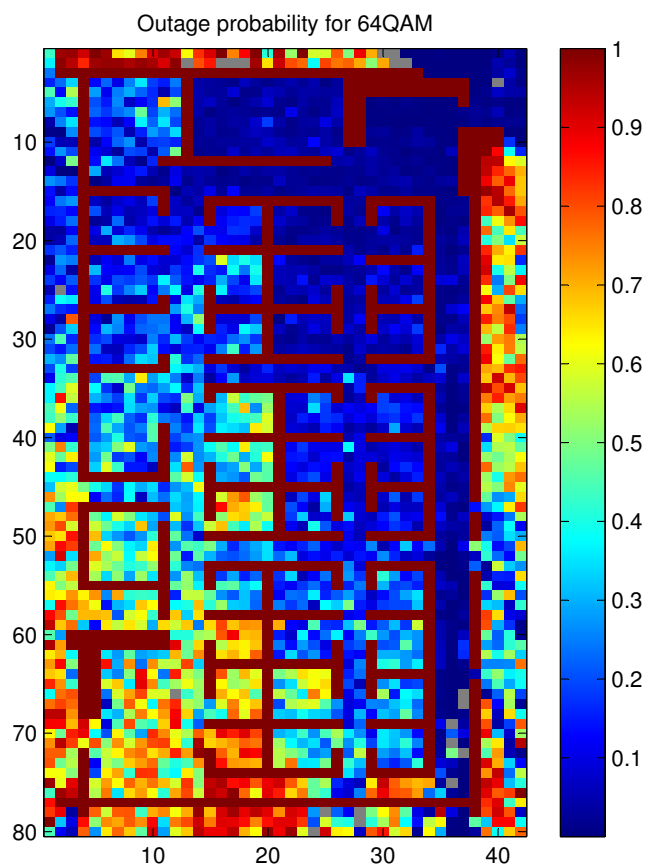

Fig. 6. Outage probability for 64QAM.

\section{Conclusion}

In this paper, a realistic $m$ parameter map of the Nakagami$\mathrm{m}$ fading and the BER map are first predicted based on the deterministic MR-FDPF model. After the error between the MR-FDPF model and the channel measurement is modeled to follow the normal distribution in $\mathrm{dB}$, the confidence interval of the predicted BER is evaluated. At the last part of the paper, the normally distributed error in $\mathrm{dB}$ between the MR-FDPF model and the channel measurement is regarded as the lognormal shadowing introduced, for instance, by the inaccurate scenario modeling. Then a realistic outage probability map is predicted by taking into account both the large scale shadow fading and the small scale Nakagami-m fading. The prediction of the radio link quality parameters in this paper can be very useful for wireless communication systems.

\section{ACKNOWLEDGMENT}

This work is funded by the FP7 IPLAN Project.

\section{REFERENCES}

[1] A. Goldsmith, Wireless communications. Cambridge university press, 2005.

[2] K. W. Sowerby, "Outage probability in mobile radio systems," Ph.D. dissertation, ResearchSpace@ Auckland, 1989.

[3] J.-M. Gorce, K. Jaffres-Runser, and G. de la Roche, "Deterministic approach for fast simulations of indoor radio wave propagation," IEEE Transactions on Antennas and Propagation, vol. 55, no. 3, pp. 938 -948, 2007.

[4] K. Runser and J.-M. Gorce, "Assessment of a new indoor propagation prediction method based on a multi-resolution algorithm," in 61st IEEE Vehicular Technology Conference, Stockholm, Sweden, May 2005.

[5] M. Luo, D. Umansky, G. Villemaud, M. Lafort, and J.-M. Gorce, "Estimating channel fading statistics based on radio wave propagation predicted with deterministic MR-FDPF method," in 5th European Conference on Antennas and Propagation (EuCAP 2011), Rome, Italy, April 2011.

[6] M. Luo, G. Villemaud, J. Gorce, and J. Zhang, "Realistic prediction of BER and AMC for indoor wireless transmissions," IEEE Antennas and Wireless Propagation Letters, vol. 11, pp. 1084 - 1087, 2012.

[7] M. Luo, G. Villemaud, J.Weng, J.-M. Gorce, and J. Zhang, "Realistic prediction of BER and AMC with MRC diversity for indoor wireless transmissions," in IEEE Wireless Communications and Networking Conference (WCNC 2013), Shanghai, China, April, 2013.

[8] N. Czink, B. Bandemer, G. Vazquez-Vilar, A. Paulraj, and L. Jalloul, "July 2008 radio measurement campaign: Measurement documentation," Stanford University, Smart Antennas Research Group, Tech. Rep., July 2008.

[9] "Rusk medav channel sounders," 2008, [Online]. Available: http://www.channelsounder.de.

[10] M. Nakagami, "The m-distribution-A general formula of intensity distribution of rapid fading," Statistical Method of Radio Propagation, 1960.

[11] Q. Zhang, "A note on the estimation of nakagami-m fading parameter," IEEE Communications Letters, vol. 6, no. 6, pp. 237-238, 2002.

[12] R. D'Agostino and M. Stephens, Goodness-of-fit techniques. New York: Marcel Dekker, 1986, vol. 68.

[13] M. Simon and M. Alouini, Digital communication over fading channels. Wiley-IEEE Press, 2004, vol. 86.

[14] S. M. Ross, Introduction to probability and statistics for engineers and scientists. Academic Press, 2009.

[15] P. Shankar, "Outage probabilities in shadowed fading channels using a compound statistical model," IEE Proceedings-Communications, vol. 152, pp. 828-832, 2005.

[16] MATLAB. Version 7.8, Release 21, (The MathWorks, Natick, MA 200). 